\title{
A geometrical argument for a theorem of G. E. Welters
}

\author{
Giambattista Marini \\ Dipartimento di matematica, II Università di Roma "Tor Vergata", Via della Ricerca \\ Scientifica, I-00133 Roma, Italy (e-mail: marini@axp.mat.uniroma2.it)
}

Received February 18, 1998; in final form August 10, 1998

The existence of a one parameter family of trisecants to the Kummer variety of an indecomposable principally polarized abelian variety characterizes Jacobians. This result was first proved by Gunning in [G] under additional hypotheses. Then Welters removed the additional hypotheses and considered the degenerate cases, see [W1] and [W2]. In this note we provide a short geometrical argument for the inflectionary case.

Theorem (Welters). Let $(X,[\Theta])$ be an indecomposable principally polarized abelian variety. Let $Y$ be the Artinian subscheme of $X$ of length 3 supported at 0 defined as the image of Spec $\mathbb{C}[\epsilon] /\left(\epsilon^{3}\right)$ in $X$ via the immersion $\epsilon \mapsto \epsilon 2 D_{1}+\epsilon^{2} 2 D_{2}$, where $D_{1} \neq 0$. Denote by $K: X \rightarrow|2 \Theta|$ the Kummer morphism. Assume that the algebraic subset

$$
V=\left\{2 u \in X \mid u+Y \subset K^{-1}(l) \text { for some line } l \subset|2 \Theta|\right\},
$$

has positive dimension. Then $V$ is a smooth irreducible curve through zero, and $(X,[\Theta])$ is the polarized Jacobian of $V$.

The proof of this theorem is naturally divided in two steps. First one proves that the endomorphism $\delta$ associated with the pair $(\mathcal{C}, \Theta)$ is a positive multiple of the identity; here $\mathcal{C}$ is any curve contained in $V$. Then one proves that $\delta$ is the identity over the flow generated by $D_{1}$ (thus $\delta$ is the identity and Matsusaka's criterion applies, moreover $\mathcal{C}=V$ by curve theory). For the first step we go back to Welters' homological argument in [W1]. For the second one we present a geometrical argument. We recall that $\delta(x)$ is, by definition, the sum in $X$ of the points of the intersection $\mathcal{C} . \Theta_{x}$, opportunely translated in order to have $\delta(0)=0$ (namely $\delta(x)=\operatorname{sum} \mathcal{C} .\left(\Theta_{x}-\Theta\right)$ ). We shall use the following remark. 
1 Remark. Assume that $V$ and $W$ are subvarieties of $X$ of complementary dimensions and assume that they intersect transversally at distinct points $p_{1}, \ldots, p_{r}$. If a point $x$ is in a small analytic neighborhood of zero, we are allowed to number the points of the intersection $V . W_{x}$ according to the numbering of the $p_{i}$ 's. Thus, we can single out the contribution of $p_{i}$ to the differential $d \delta$. We shall denote this contribution by $d \delta_{p_{i}}$. Let $D$ be a vector in $T_{0}(X)$, or better an invariant vector field on $X$. We claim that, for $d \delta_{p_{i}}(D)$, which is the $i^{t h}$-contribution to $\lim _{\epsilon \rightarrow 0} \frac{1}{\epsilon} \operatorname{sum}\left(V .\left(W_{\epsilon D}-W\right)\right)$, the following holds. If $D$ is tangent to $V$ at $p_{i}$, then $d \delta_{p_{i}}(D)=D$. If $D$ is tangent to $W$ at $p_{i}$, then $d \delta_{p_{i}}(D)=0$. Our claim is a trivial consequence of the fact that since $V$ and $W$ intersect transversally at $p_{i}$, for the computation of the contribution $\delta_{p_{i}}$ up to its first order, we are allowed to work with tangent spaces.

Let us go back to the hypothesis of the theorem. The image under $K$ of the germ of curve $u+Y=u+2 \epsilon D_{1}+2 \epsilon^{2} D_{2}$ is the germ of curve $\vec{\vartheta}(u+$ $\left.2 \epsilon D_{1}+2 \epsilon^{2} D_{2}\right)=\vec{\vartheta}(u)+2 \epsilon D_{1} \vec{\vartheta}(u)+2 \epsilon^{2}\left(D_{1}^{2}+D_{2}\right) \vec{\vartheta}(u) \bmod \left(\epsilon^{3}\right)$, where $\vec{\vartheta}:=\left(\ldots, \vartheta_{\sigma}, \ldots\right),\left\{\vartheta_{\sigma}\right\}$ is a basis of $H^{0}(X, 2 \Theta)$. The image germ above, viewed in $\mathbb{P} H^{0}(X, 2 \Theta)$, is contained in a straight line if and only if the three terms of degrre 0,1 and 2 are dependent, thus

$2 u \in V \quad$ if and only if $\operatorname{rank}\left(\vec{\vartheta}(u) D_{1} \vec{\vartheta}(u)\left(D_{1}^{2}+D_{2}\right) \vec{\vartheta}(u)\right) \leq 2$.

Observe that $V$ parametrizes inflectionary points of the Kummer variety. Also, we recall that by a well know degeneration of Fay's trisecant formula (e.g. see [W1]),

(*) $x \in V, x \neq 0 \quad$ if and only if $\quad \Theta \cap \Theta_{x} \subset D_{1} \Theta \cup D_{1} \Theta_{x}$, where $D_{1} \Theta:=\Theta \cap\left\{D_{1} \theta=0\right\}$, and $\theta$ is a generator of $H^{0}(X, \Theta)$.

2 Remark (see [W2]). If $V$ has positive dimension at 0 , then $V$ is smooth, one-dimensional at 0 . Furthermore $T_{0}(V)=\left\langle D_{1}\right\rangle$. To prove this claim, first observe that the condition

$$
\operatorname{rank}\left(\vec{\vartheta}(u) D_{1} \vec{\vartheta}(u)\left(D_{1}^{2}+D_{2}\right) \vec{\vartheta}(u)\right) \leq 2
$$

gives rise to natural determinantal equations defining $V$ as a scheme. These equations are the determinants equated to zero of the minors of order 3 of the matrix above. For computational purpose it is convenient to work with $\Lambda^{3} H^{0}(X, 2 \Theta)$, so that

$$
V=\left\{2 u \mid f(u):=\vec{\vartheta}(u) \wedge D_{1} \vec{\vartheta}(u) \wedge\left(D_{1}^{2}+D_{2}\right) \vec{\vartheta}(u)=0\right\} .
$$

Furthermore, once for all we recall that $\overrightarrow{\mathcal{D} \vartheta}(0)=0$ whenever $\mathcal{D}$ is a derivative of odd order. Note that, in particular, $f(0)=0$, i.e. $0 \in V$. 
As $E \in T_{0}(V)$ if and only if $E f(0)=0$, to prove the claim it suffices to show that

$$
E f(0)=0 \quad \text { if and only if } \quad E \in\left\langle D_{1}\right\rangle .
$$

We have $E f(0)=E\left(\vec{\vartheta} \wedge D_{1} \vec{\vartheta} \wedge\left(D_{1}^{2}+D_{2}\right) \vec{\vartheta}\right)(0)=\vec{\vartheta}(0) \wedge E D_{1} \vec{\vartheta}(0)$ $\wedge D_{1}^{2} \vec{\vartheta}(0)$ which equals zero if and only if $E \in\left\langle D_{1}\right\rangle$ by Wirtinger's theorem stating that the matrix $\left(\vec{\vartheta}(0), \ldots, E_{i} E_{j} \vec{\vartheta}(0), \ldots\right)$ has maximal rank $1+\frac{1}{2} n(n+1)$, where $n:=\operatorname{dim}(X)$ and $\left\{E_{1}, \ldots, E_{n}\right\}$ is a basis of $T_{0}(X)$, provided that $(X,[\Theta])$ is indecomposable. Thus we are done. We now come to the proof of the theorem.

Proof (of the Theorem). Let $\mathcal{C}$ be an irreducible (and reduced) curve contained in $V$. We want to prove that the endomorphism $\delta$ is the identity. By hypothesis, the following inclusions hold

$$
\Theta \cap \Theta_{x} \subset D_{1} \Theta \cup D_{1} \Theta_{x}, \quad \forall x \in V, x \neq 0 .
$$

Step 1. The endomorphism $\delta$ is a multiple of the identity.

This follows by Welters' homological argument (see [W1], p. 503), which we now recall for the sake of completeness. By the inclusions above there exist algebraic cycles of codimension 2 in $X, M^{\prime}$ and $M$, such that

$$
\Theta \cap \Theta_{x}=M^{\prime}+M_{x}, \quad \forall x \in \mathcal{C}, x \neq 0 .
$$

The $M_{x}$ 's cover $\Theta$ as $x$ moves in $\mathcal{C}$. It follows that the Pontryagin product of homology classes $[M] \star[\mathcal{C}]$ is $c \cdot[\Theta]$, where $c$ is a constant. On the other hand, since $\Theta_{-x} \cap \Theta=M_{-x}^{\prime}+M$ and since the cycle $-\mathcal{C}$ is homologically equivalent to the cycle $\mathcal{C}$, there exists a constant $c^{\prime}$ such that the Pontryagin product $\left[M^{\prime}\right] \star[\mathcal{C}]$ equals $c^{\prime} \cdot[\Theta]$. Thus $\left[\Theta^{2}\right] \star \mathcal{C}=$ $\left[M^{\prime}+M\right] \star[\mathcal{C}]=\left(c^{\prime}+c\right) \cdot[\Theta]=$ const $\left[\Theta^{2}\right] \star\left[\Theta^{n-1}\right] /(n-1) !$. As $\Theta$ is ample, the map $\left(\left[\Theta^{2}\right] \star\right): H_{2}(X) \longrightarrow H_{2 n-2}(X)$ is an isomorphism. It follows that $[\mathcal{C}]=\operatorname{const}\left[\Theta^{n-1}\right] /(n-1)$ !, so that the endomorphism $\delta$ is a multiple of the identity as claimed. This multiple must be positive because $\mathcal{C}$ is effective and $\Theta$ is ample.

Step 2. The differential $d \delta$ sends the vector field $D_{1}$ to itself.

Both the inclusions $(\star)$ and the endomorphism $\delta$ are independent on the translate of the theta divisor we choose. Denote by $D_{1} \mathcal{C}$ the set of points $p$ of $\mathcal{C}$ such that $D_{1}$ is tangent to $\mathcal{C}$ at $p$ (in particular $D_{1} \mathcal{C}$ includes singular points of $\mathcal{C}$ ). Note that $D_{1} \mathcal{C}$ is finite. Otherwise, the curve $\mathcal{C}$ would be $D_{1}$-invariant, thus it would be an elliptic curve, and the image of $\delta$ would be contained in the translate of $\mathcal{C}$ through 0 , contradicting that $\delta$ is a non-zero multiple of the identity. Modulo translations of $\Theta$ we can assume the following: 
1) $\mathcal{C} \not \subset \Theta$

2) $0 \in \Theta_{\text {smooth }}$;

3) $D_{1} \notin T_{0}(\Theta)$;

4) $\mathcal{C} \cap \Theta_{\text {sing }}$ is empty;

5) $D_{1} \mathcal{C} \cap D_{1} \Theta$ is empty.

Indeed, in order to find a translate $\tilde{\Theta}:=\Theta_{-x}$ satisfying $1,2,3,4,5$, it suffices to require that the point $x$ is in

$$
\begin{aligned}
& \Theta_{\text {smooth }}-\left(\Delta\left(\left(\left(\{0\} \cup D_{1} \mathcal{C}\right) \times D_{1} \Theta\right) \cup\left(\mathcal{C} \times \Theta_{\text {sing }}\right)\right)\right. \\
& \left.\cup\left\{x \in X \mid \mathcal{C} \subset \Theta_{-x}\right\}\right),
\end{aligned}
$$

where $\Delta$ is the difference map $\mathcal{C} \times \Theta \rightarrow X,(p . q) \mapsto q-p$. We claim that the set above is not empty. The set $\Delta\left(\left(\left(\{0\} \cup D_{1} \mathcal{C}\right) \times D_{1} \Theta\right) \cup\left(\mathcal{C} \times \Theta_{\text {sing }}\right)\right)$ has at most dimension $n-2$, thus it does not contain $\Theta_{\text {smooth }}$ (here we use Ein-Lazarsfeld's theorem on Arbarello-De Concini's conjecture stating that $\operatorname{dim} \Theta_{\text {sing }} \leq n-3$, cf. [EL]). If the set $A:=\left\{x \in X \mid \mathcal{C} \subset \Theta_{-x}\right\}$ has dimension $n-1$, the inverse image $\Delta^{-1}(A)$ is $\mathcal{C} \times \Theta$. In fact, the product $\mathcal{C} \times \Theta$ is irreducible and the fiber $\Delta^{-1}(x)$ has positive dimension for all $x \in A$ (as $\mathcal{C} \subset \Theta_{-x}, \mathcal{C}+x \subset \Theta$ and $\Delta^{-1}(x)$ is the set of pairs $\left.(p, p+x)_{p \in \mathcal{C}}\right)$. As a consequence, if $\Theta_{-x}$ meets $\mathcal{C}$, then it contains $\mathcal{C}$. This leads to a contradiction. In fact, it would imply that the general translate of $\Theta$ does not meet $\mathcal{C}$. This concludes the proof of our claim.

By remark 2, if $\mathcal{C}$ contains zero, then it is smooth at zero and $D_{1} \in$ $T_{0}(\mathcal{C})$. It follows that if $0 \in \mathcal{C}$, the theta divisor meets $\mathcal{C}$ transversally at zero by the $3^{\text {th }}$ condition. Set now $\left\{x_{1}, \ldots, x_{r}\right\}:=\Theta \cap(\mathcal{C}-\{0\})$. As 0 and $x_{i}$ are in $\Theta$, the point $x_{i}$ is in the left hand side of the inclusion $\Theta \cap \Theta_{x_{i}} \subset D_{1} \Theta \cup D_{1} \Theta_{x_{i}}$, so that it must be in the right hand side. This implies that either $D_{1}$ is in the tangent space $T_{x_{i}}(\Theta)$, or $D_{1}$ is in the tangent space $T_{0}(\Theta)$. The latter case does not occur by the $3^{\text {th }}$ condition, thus $D_{1}$ is in the tangent space $T_{x_{i}}(\Theta)$ for all $i$. On the other hand, by the $5^{\text {th }}$ condition, the intersection $D_{1} \mathcal{C} \cap D_{1} \Theta$ is empty, so that $D_{1} \notin T_{x_{i}}(\mathcal{C})$. In particular, $\Theta$ and $\mathcal{C}$ meet transversally at the $x_{i}$ 's, hence they meet transversally (recall that if $0 \in \mathcal{C}$, then $\Theta$ and $\mathcal{C}$ meet transversally at zero). We now apply remark 1 . Since $D_{1}$ is in the tangent space $T_{x_{i}}(\Theta)$ for all $i$, then $d \delta_{x_{i}}\left(D_{1}\right)=0$ for all $i \quad\left(d \delta_{x_{i}}\right.$ has been introduced in remark 1). If $\mathcal{C}$ does not contain 0 , it would follow $d \delta\left(D_{1}\right)=0$. This is impossible because, by the first step, $d \delta\left(D_{1}\right)$ is a positive multiple of $D_{1}$. On the other hand, again by remark $1, d \delta_{0}\left(D_{1}\right)$ equals $D_{1}$ because $D_{1}$ is tangent to $\mathcal{C}$ at zero, and it is transverse to the tangent space $T_{0}(\Theta)$. Taking the sum over all points of the intersection $\mathcal{C} . \Theta$ we obtain that the image of $D_{1}$ under the differential $d \delta$ is $D_{1}$ as claimed.

q.e.d. 


\section{References}

[EL] L. Ein, R. Lazarsfeld, Singularities of theta divisors, and the birational geometry of irregular varieties, Preprint (alg-geom/9603017)

[G] R. C. Gunning, Some curves in abelian varieties, Invent. Math. 66 (1982), 377-389

[W1] G. E. Welters, A criterion for Jacobi varieties, Ann. Math. 120 (1984), 497-504

[W2] G. E. Welters, On flexes of the Kummer variety, Indagationes Math. 45 (1983), 501520 